\title{
Therapeutic and Analgesic Efficacy of Laser in Conjunction With Pharmaceutical Therapy for Trigeminal Neuralgia
}

\author{
Hooman Ebrahimi ${ }^{1}$, Shamsolmoulouk Najafi ${ }^{2}$, Mina Khayamzadeh², Amirabbas Zahedi ${ }^{3}$, Atossa Mahdavi ${ }^{4}$ \\ ${ }^{1}$ Oral Medicine Department, Dental Branch, Islamic Azad University, Tehran, Iran \\ ${ }^{2}$ Oral Medicine Department, Dental Research Center, Faculty of Dentistry, International Campus of Tehran University of \\ Medical Sciences, Tehran, Iran \\ ${ }^{3}$ Faculty of Dentistry, International Campus of Tehran University of Medical Sciences, Tehran, Iran \\ ${ }^{4}$ Department of Obstetrics and Gynecology, Shariati Hospital, Tehran University of Medical Sciences, Tehran, Iran
}

\section{*Correspondence to Shamsolmoulouk Najafi, Associate Professor of Oral medicine Department, Dental of School, International Campus, Tehran University of Medical Sciences, Tehran, Iran. Tel: +98 9123236378; Fax: +98 2155845425 Email: Najafi_sh@tums.ac.ir}

Published online 26 December 2017

\begin{abstract}
Introduction: Trigeminal neuralgia (TN) is the most common neuralgia in the head and neck region and a common cause of orofacial pain. It is routinely treated with carbamazepine. Laser, acupuncture and radiofrequency are among other treatment modalities for this condition. This study sought to assess the efficacy of laser therapy in conjunction with carbamazepine for treatment of TN.

Methods: A total of 30 patients who met the inclusion criteria were divided into 2 groups of cases and controls $(n=15)$ by double blind randomized controlled clinical trial. All patients received 100 mg carbamazepine at baseline and another $100 \mathrm{mg}$ after 2 days for pain control. In the case group, low level laser therapy (LLLT) was also performed in addition to pharmaceutical therapy. Sham laser was used in the control group instead of LLLT. Treatment was continued for 9 sessions (3 days a week). The intensity of pain was measured and compared in the 2 groups using visual analog scale (VAS) in 3 period. The qualitative variables among the groups were compared using the repeated measures analysis of variance (ANOVA).

Results: The severity of pain was lower at the end of treatment in the case compared to the control group so this difference was statistically significant $(P=0.003)$. The severity of pain decreased in both groups over time. Significant difference was noted in this regard between the 2 groups either $(P=0.003)$. At the end of treatment pain intensity dropped in the intervention group from $6 / 8$ to $1 / 2$ and control group from $6 / 6$ to $2 / 7$.

Conclusion: Laser therapy did add to the value of pharmaceutical therapy for treatment of TN. Both groups experienced significant improvement over time. So it is better to used laser complementary therapy to reduce side effects and the medicine dosage.

Keywords: Trigeminal neuralgia; Carbamazepine; Laser.
\end{abstract}

\section{Introduction}

Trigeminal neuralgia (TN) is the most common neuralgia of the head and neck region and is a major cause of orofacial pain. ${ }^{1,2} \mathrm{TN}$ was first described by John Locke in 1677. Nicolas Andre later described TN by the term tic doloreaux, ${ }^{1}$ which means painful spasm. This term has also been accepted as an alternative name for $\mathrm{TN}^{1-5}$

Averagely, 4 out of 100000 individuals develop TN annually. ${ }^{1,4,6}$ Occurrence of idiopathic TN before the age of 40 years is rare. ${ }^{2}$ But it is common among the middle aged and the elderly after the age of 50 years. ${ }^{1,2,7-9}$ Moreover, TN is more common among females. ${ }^{4,5,7,10}$ Pain in TN is acute and striking, with sudden onset and termination. It is often described to be similar to electric shock, perforating, penetrating, superficial and sharp. ${ }^{3,6,8-10}$

Pain strikes may be spontaneous or triggered. ${ }^{1-4}$ Most patients, but not all, have a trigger zone. Trigger zone is pathognomonic for TN. Most common sites include perinasal skin, peri-oral skin, lips, nasolabial fold, gingiva and alveoli. Other areas such as cheeks, eyebrows, periorbital skin and tongue may also serve as trigger zones. Most patients localize their trigger zone and avoid its stimulation. ${ }^{4-7,10,11}$ Stimulation often occurs by contact (skin and mucosa) and less commonly by movement. ${ }^{1,5}$ Pain is often unilateral ${ }^{3,8,9,12}$ and does not cross the midline. Pathogenesis of TN is not well understood..$^{1-3}$ Vascular pressure is the main cause of demyelination of nerves compressing the trigeminal nerve when entering the

Please cite this article as follows: Ebrahimi $\mathrm{H}$, Najafi S, Khayamzadeh M, Mahdavi A. Therapeutic and analgesic efficacy of laser in conjunction with pharmaceutical therapy for trigeminal neuralgia. J Lasers Med Sci. 2018;9(1):63-68. doi:10.15171/jlms.2018.13. 
pons. ${ }^{1,3-5}$ This is where peripheral nervous system myelin sheath is terminated and central nervous system myelin sheath covers the nerves. ${ }^{1}$ Since TN is severely painful, accurate diagnosis and prompt treatment are of utmost importance. Diagnosis of TN is based on taking a precise history of the pain attacks. Also, evaluation of history is essential to rule out other causes of orofacial pain. ${ }^{4}$

There is no diagnostic test for definite diagnosis of TN. Even magnetic resonance imaging cannot definitely confirm the diagnosis of TN. ${ }^{1-3}$ Although radiography and referral for specific tests for other conditions may be required to rule out differential diagnoses, diagnosis of $\mathrm{TN}$ is completely clinical ${ }^{4}$ and interview with the patient and a thorough clinical examination will often suffice to make a diagnosis.

Treatment of TN is primarily pharmaceutical., ${ }^{2,4,13,14}$ Clinically, it has been documented that anticonvulsant drugs are effective for alleviation of neuropathic pains especially those described as burning or perforating pain. Thus, anticonvulsant drugs are mainly prescribed for TN.? Use of carbamazepine to alleviate pain due to $\mathrm{TN}$ has a long history. Many controlled and uncontrolled clinical trials and meta-analyses support prescription of carbamazepine as the first line treatment for TN.,4,8,15 Thus, carbamazepine is considered as the gold standard first line treatment for TN. ${ }^{3,7,11,16,17}$ In therapeutic doses, carbamazepine blocks the sodium channels and decreases the synaptic transmission. ${ }^{5,11}$ Carbamazepine has side effects such as imbalance, ${ }^{5,11}$ nausea, ${ }^{3,11,15}$ sleepiness, ${ }^{3,11}$ confusion, ${ }^{5}$ dizziness ${ }^{11,13}$ and loss of appetite., ${ }^{3,11}$ In $10 \%$ of patients, transient leukopenia ${ }^{15,18}$ and thrombocytopenia ${ }^{15,18}$ may occur. Patients who have low level of white blood cells prior to treatment are at higher risk of developing leukopenia. ${ }^{15,18}$

Low level lasers decrease acute and chronic pains via different mechanisms such as decreasing the level of histamine, acetyl choline, serotonin, bradykinin and prostaglandin E2, increasing acetyl choline esterase, lymphatic drainage, ATP, aerobic metabolism, pain threshold, beta-endorphins and enkephalins, balancing the activity of adrenaline- noradrenaline and decreasing the production of $\mathrm{P}$ substance in the posterior spinal horn. ${ }^{14,17,19-21}$

Laser irradiation dilates the capillaries and increases the drainage of fluids in the interstitial space by stimulating their reuptake. It exerts its analgesic effect by breaking the vicious cycle of contraction-edema-pain. Electromagnetic laser energy increases the metabolism of the injured tissue. This effect is enhanced by increased production of ATP and repolarization of cell membrane. Laser decreases the release of substances that stimulate pain receptors and significantly increases the pain threshold by stimulating the synthesis of endorphins. ${ }^{22}$ This study aimed to compare the severity of pain in patients with $\mathrm{TN}$ under treatment with carbamazepine with and without laser irradiation.

\section{Methods}

This double blind randomized controlled clinical trial was conducted on 30 patients with TN. Sample size was calculated to be 30 patients ( $n=15$ in each group) according to a previous study by Amanat et al in 2013. ${ }^{23}$ The patients were selected among those presenting to Laser Center of Pardis International Campus, Tehran University of Medical Sciences.

The inclusion criteria: Patients with definite diagnosis of $\mathrm{TN}$ according to the criteria of the International Headache Society (HIS) were included [HIS 3 with ICD10 (G44.0) coding]. Patients who were under standard pharmaceutical treatment for TN but had not experienced any improvement were also included.

The exclusion criterion was pregnancy and patients with abnormal $\mathrm{CBC}$ and liver function tests. Patients were divided into 2 groups of cases and controls using block randomization. After the intervention in the 2 groups, the efficacy of treatment in terms of change in the severity of pain was assessed using the visual analog scale (VAS). The severity of pain was recorded prior to laser therapy, immediately after each session of laser therapy and one month after completion of treatment using the VAS. The pain scores were recorded in a questionnaire. The VAS ranged from $0-10 ; 10$ indicated the most severe pain imaginable while 0 indicated no pain. Laser treatment was performed for 9 sessions, 3 days a week.

Laser therapy was performed at the trigger points, based on previous studies we use laser and carbamazepine in case groups for treatment of TN. GaAlAs diode laser was irradiated at $810 \mathrm{~nm}$ wavelength with $5 \mathrm{~J}$ energy in continuous mode during 9 sessions using Thor device. The probe tip of the device was $1 \mathrm{~cm}$ in diameter and laser was irradiated for 25 seconds with maximum output power of $200 \mathrm{~mW}$. If trigger point was not found, 2 to 3 points were chosen in the path of pain indicated by patient and irradiated with laser. Laser therapy was performed by a technician. The statistical consultant was double blinded to the type of laser used for patients in the case group. Due to ethical considerations, patients in both groups were subjected to standard pharmaceutical therapy with $100 \mathrm{mg}$ carbamazepine at the onset of treatment and then another $100 \mathrm{mg}$ after 2 days based on severity of pain in patients. This process was continued until pain was alleviated taking into account the maximum safe dose of carbamazepine. Low level laser therapy (LLLT) along with pharmaceutical therapy was performed for the case group. Sham laser was used instead of LLLT in the control group.

Probe of the device was disinfected and covered with disposable covers prior to intraoral or extra oral use. For use of sham laser in the control group, the device was turned on but irradiation was not started (the start button was off). Since the device does not irradiate any visible light and has no pointer, the patients could not find out whether the device was on or off. 
Assessment of the process of change in pain severity prior to treatment, at the end of treatment and one month after completion of treatment was done using repeated measures analysis of variance (ANOVA) with time of assessment as within subject factor and type of intervention (laser or sham laser) as between subject factor.

\section{Results}

A total of 30 patients participated in this study in 2 groups of $15 ; 36.67 \%$ were males and $63.33 \%$ were females (there were $33.33 \%$ males and $66.66 \%$ females in the case group and $40 \%$ males and $60 \%$ females in the control group) (Table 1).

Pain severity in the 2 groups decreased with time (until the end of treatment) and this reduction was statistically significant $(P=0.003)$. Pain recurred at 4 months following completion of treatment and this change in pain score was statistically significant $(P=0.003$, Table 2 , Figure 1). In general, this difference was significant in any of the time points. The trend of change in pain score was significantly different between the 2 groups

Although the severity of pain in the case group was less than that in the control group. The difference between the 2 groups was significant at any time point significant difference was noted in the trend of change in pain score between the 2 groups either.

\section{Discussion}

Low level diode laser is extensively used for treatment of wounds, inflammation and chronic pain. Laser therapy was used in this study due to its analgesic, antiinflammatory and biological properties. Laser therapy regulates the blood flow and exerts its analgesic effects by decreasing the spasm of arterial muscles, which is required to enhance blood supply to the area. It increases the production of ATP and regulates the basic metabolism of tissues with limited source of energy to enhance oxygenation of hypoxic cells in trigger points. Another suggested mechanism for laser is its effect on the

Table 1. Number (percentage) of Male and Female Patients in the 2 Groups of Cases and Control

\begin{tabular}{lcc}
\hline Group & Male & Female \\
\hline Case & $5(33.33 \%)$ & $10(66.67 \%)$ \\
Control & $6(40 \%)$ & $9(60 \%)$ \\
Total & $11(36.67 \%)$ & $19(63.33 \%)$ \\
\hline
\end{tabular}

Table 2. The Mean $( \pm$ Standard Deviation) Pain Score in the 2 Groups at Different Time Points

\begin{tabular}{lcc}
\hline & \multicolumn{2}{c}{ Time of Assessment } \\
\cline { 2 - 3 } & $\begin{array}{c}\text { Upon Completion of } \\
\text { Treatment }\end{array}$ & $\begin{array}{c}\text { At 1 Month After } \\
\text { Completion of Treatment }\end{array}$ \\
\hline Case & $2.700 .49 \pm$ & $3.600 .39 \pm$ \\
Control & $2.100 .51 \pm$ & $3.200 .49 \pm$ \\
\hline
\end{tabular}

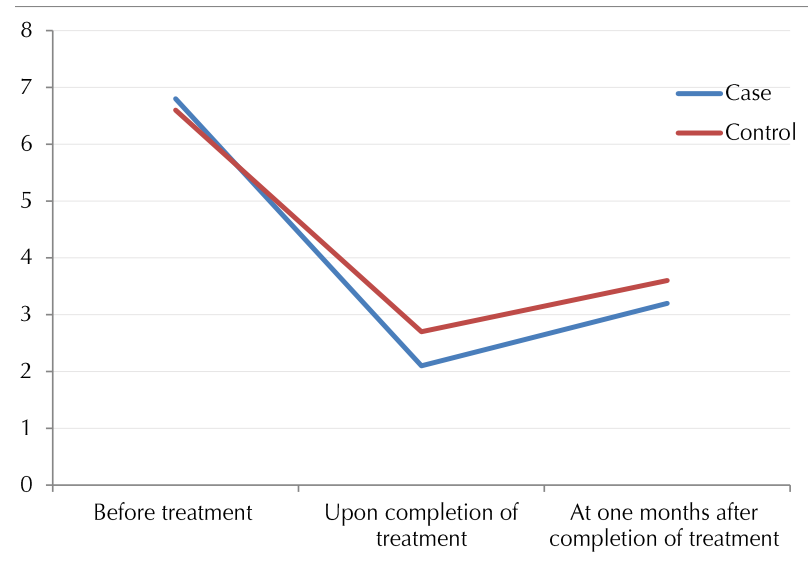

Figure 1. The Mean Severity of Pain in the 2 Groups at Different Time Points.

level of endorphins and subsequent pain control. Laser increases the level of endorphins and decreasing the level of serotonin.

Only limited studies with case-control design have evaluated the effects of laser therapy on TN with controversial results. A few studies reported positive efficacy of laser therapy for treatment of TN while some others found no significant difference between laser and control groups. Based on these differences and suggestions regarding selection of particular wavelengths, sample size and blinding, this study was conducted to further evaluate this topic.

In the current study, GaAlAs laser with $810 \mathrm{~nm}$ wavelength and $6.36 \mathrm{~J} / \mathrm{cm}^{2}$ dose in continuous mode was used for 9 sessions ( 3 sessions per week). Irradiation time was 25 seconds and maximum power of device was $200 \mathrm{~mW}$. Analgesic effects of laser in case group compared with control group was statistically significant. Our findings were not in line with those of Öz et al, ${ }^{24}$ Dundar et $\mathrm{al},{ }^{25}$ Altan et $\mathrm{al}^{26}$ and Hansen et al. ${ }^{27}$ The afore mentioned studies used different wavelengths and powers of laser and had different sample sizes and variable number of treatment sessions. However, they all had a case control design and yielded similar results. They all found no significant difference between the laser and control groups in terms of pain relief. A meta-analysis conducted by Fulop et al, ${ }^{28}$ reported the same results.

Öz et $\mathrm{al}^{24}$ treated 20 patients with myofascial pain by $820 \mathrm{~nm}$ low level diode laser with $3 \mathrm{~J} / \mathrm{cm}^{2}$ energy density and $300 \mathrm{~mW}$ power twice a week (10 sessions). The control patients received an occlusal splint for 24 hours a day for 3 months. No significant difference was noted between the 2 groups and laser therapy was as effective as occlusal splint for treatment of myofascial pain. ${ }^{24}$ Hansen et $\mathrm{al}^{27}$ evaluated the efficacy of treatment of patients with different types of orofacial pain (oral dysesthesia, toothache, TN, TN secondary to trauma and tension headache) with diode infrared laser at $904 \mathrm{~nm}$ wavelength (OKA laser). In their double blind randomized controlled 
trial, laser with a constant or variable pulse repetition rate of 1 to $9999 \mathrm{~Hz}$ with $78.9 \mathrm{~mW} / \mathrm{cm}^{2}$ power was used. Laser treatment was performed with $4.7 \mathrm{~J} / \mathrm{cm}^{2}$ energy for 60 seconds in the first 2 sessions. In case of no pain relief, laser was irradiated with $9.4 \mathrm{~J} / \mathrm{cm}^{2}$ energy for 120 seconds in the next sessions. Treatment was continued for eight sessions during four weeks. No statistically significant difference was noted between the laser and sham laser groups.

It should be noted that all the afore mentioned studies reported positive efficacy of laser therapy in decreasing the pain score (according to the VAS), which was also in line with our findings.

Some previous studies did not mention the energy density of laser used. Some others chose energy densities over 20 $\mathrm{J} / \mathrm{cm}^{2}$ or lower than $5 \mathrm{~J} / \mathrm{cm}^{2}$. Only a few studies chose a moderate energy density. Thus, we used $6.36 \mathrm{~J} / \mathrm{cm}^{2}$ energy density. Carrasco et $\mathrm{al}^{29}$ used very high energy densities $\left(25,60,105 \mathrm{~J} / \mathrm{cm}^{2}\right)$. Since they did not obtain favorable results and based on the suggested energy density values for GaAsAl laser, ${ }^{30}$ we used a lower energy density. In the study by $\mathrm{O} z$ et al, ${ }^{24} 3 \mathrm{~J} / \mathrm{cm}^{2}$ and in the study by Hansen et $\mathrm{al}^{27} 4.7$ and $9.4 \mathrm{~J} / \mathrm{cm}^{2}$ energy densities were chosen. Based on their findings, we selected a higher energy density.

To assess the stability of the results of laser therapy, VAS has been used in previous studies. Carrasco et $\mathrm{al}^{29}$ assessed the level of pain of patients using a VAS 15 days and 1 month following completion of treatment. Dundar et $\mathrm{al}^{25}$ used VAS 4 weeks after initiation of treatment and Altan et $\mathrm{al}^{26}$ used VAS at $0,2,12$ and 14 weeks following treatment to assess the pain severity in patients. Despite variability in assessment time points, similar results were reported. In the current study, a slight increase in pain scores of patients (based on VAS) was noted after completion of treatment and one month after completion of treatment in the 2 groups and significant difference was noted. The laser therapy protocol was twice a week for 10 sessions by Öz et al, ${ }^{24}$ twice a week for 4 weeks by Carrasco et al, ${ }^{29}$ once a day for 15 days by Dundar et al, ${ }^{25}$ once a day for 10 days by Altan et al, ${ }^{26}$ and 8 sessions during 4 weeks by Hansen et al. ${ }^{28}$ Considering the fact that the effects of each session of laser therapy often last for nine to 72 hours and also to prevent the cumulative effects of laser, we performed laser therapy for 3 sessions a week.

Since $\mathrm{HeNe}$ and GaAs laser was used in some previous studies with no significant difference between the case and control groups, GaAlAs laser was used in the current study.

Carrasco et $\mathrm{al}^{29}$ Shirani et $\mathrm{al},{ }^{31}$ Hakgüder et $\mathrm{al},{ }^{32}$ and Walker ${ }^{33}$ reported results that were similar to ours. They found significant differences between the case and control groups and reported superior efficacy of laser compared to the placebo. Carrasco et $\mathrm{al}^{29}$ treated patients with myofascial pain using $780 \mathrm{~nm}$ GaAlAs laser with 25, 60 and $105 \mathrm{~J} / \mathrm{cm}^{2}$ density in continuous mode twice a week for 4 weeks. Control groups were subjected to placebo laser therapy. A significant reduction in pain was noted in both groups with significant difference between the 2 $(P<0.001)$.

These studies used a variety of laser types, densities, sample sizes and irradiation protocols.

Walker ${ }^{33}$ evaluated the efficacy of repeated radiations of low level HeNe laser for chronic pain and reported optimal pain control. Of 26 patients with TN and neuralgia following herpes infection, 19 experienced pain relief with laser without taking any analgesics. No such a result was obtained in sham laser group. Laser therapy in their study was performed for 30 sessions ( 3 times a week) with HeNe laser at $632 \mathrm{~nm}$ wavelength, $1 \mathrm{~mW}$ power and $20 \mathrm{~Hz}$ frequency for 20 seconds in an area measuring $4 \mathrm{~mm}^{2}$

Shirani et $\mathrm{al}^{31}$ used laser with $6.2 \mathrm{~J} / \mathrm{cm}^{2}$ energy density twice a week for 3 weeks while Hakgüder et al $^{32}$ used laser with $5 \mathrm{~J} / \mathrm{cm}^{2}$ energy density. GaAlAs laser was used in both studies, which was similar the laser used in our study. The selected wavelength of laser by Shirani et al, ${ }^{31}$ Hakgüder et $\mathrm{al}^{32}$ and Walker ${ }^{33}$ was within the effective wavelength range with adequate penetration depth for deep pains (650 to $1000 \mathrm{~nm}$ wavelengths have a penetration depth of $20-40 \mathrm{~mm}){ }^{30}$

Yang et $\mathrm{al}^{34}{ }^{34}$ IIbuldu et $\mathrm{al},{ }^{35}$ Pinheiro et $\mathrm{al},{ }^{36,37}$ and Gam et $\mathrm{al}^{38}$ performed interventional studies with one group. Our study was a double blind controlled clinical trial with one active control group. Due to ethical considerations, pharmaceutical therapy was performed in both groups. Sham laser was used in the control group instead of LLLT in order to eliminate the psychological effect of laser therapy as a confounding factor on the results. Yang et $\mathrm{al}^{34}$ evaluated 16 patients with idiopathic facial pains. They used $800 \mathrm{~nm}$ diode laser with $105 \mathrm{~J} / \mathrm{cm}^{2}$ energy density for 10 sessions. The VAS showed a mean reduction in pain by $43.87 \%$. Pinheiro et $\mathrm{al}^{36,37}$ evaluated the effects of diode laser at $632.8 \mathrm{~nm}, 670 \mathrm{~nm}$ and $830 \mathrm{~nm}$ wavelengths with a radiated dose of $1.8 \mathrm{~J} / \mathrm{cm}^{2}$ energy density on chronic pain such as TN, muscle pain and temporomandibular joint pain. Laser therapy was performed for 12 sessions, twice a week. The results showed optimal efficacy of laser for maxillofacial pain relief. The same group of researchers evaluated the efficacy of the same wavelengths of laser with the same protocol but with $2.5 \mathrm{~J} / \mathrm{cm}^{2}$ energy density for maxillofacial pains (temporomandibular joint pain, $\mathrm{TN}$, muscle pain, pain due to aphthous ulcer and inflammation and tooth hypersensitivity) and reported optimal analgesic efficacy of LLLT for maxillofacial pains. ${ }^{36,37} \mathrm{Gam}$ et al, ${ }^{38}$ in a meta-analysis on the efficacy of LLLT for musculoskeletal pain concluded that it had no effect on pain due to musculoskeletal syndromes.

It is difficult to reach a conclusion regarding a specific dosage, wavelength or type of laser with the highest analgesic efficacy. In most cases, minimum dosage is not known and various doses have been reported for each type of laser. Selection of the most appropriate wavelength 
is also difficult because the conclusions have been drawn mainly based on clinical experiences of the operators, and a widely accepted protocol does not exist in this regard. For instance, it has been suggested that laser therapy activates the somatosensory receptors and decreases regional pain perception, causing relaxation at the trigger points. However, this theory does not apply to deeper trigger points. Thus, variability in the results of studies may be explained by differences in laser parameters.

Further studies are required on different types, wavelengths and energy densities of laser for trigger points at different depths in patients with TN.

\section{Conflict of Interests}

The authors declare that they have no conflict of interest.

\section{Ethical Considerations}

The study protocol was approved in the ethics committee of the university (code: IR.TUMS.VCR.REC.1395.498) and registered in the Iranian Registry of Clinical Trials website (identifier: IRCT201601142464N15, http://www. irct.ir). All patients signed written informed consent forms.

\section{References}

1. Bagheri SC, Farhidvash F, Perciaccante VJ. Diagnosis and treatment of patients with trigeminal neuralgia. J Am Dent Assoc. 2004;135(12):1713-1717.

2. Aguggia M. Typical facial neuralgias. Neurol Sci. 2005;26 Suppl 2:s68-s70. doi:10.1007/s10072-005-0411-z.

3. De Leeuw R. Orofacial Pain. Chicago: Quintessence; 2008.

4. Krafft RM. Trigeminal neuralgia. Am Fam Physician. 2008;77(9):1291-1296.

5. Fauci AS. Harrison's Principles of Internal Medicine: New York, NY: McGraw-Hill; 2008.

6. Matwychuk MJ. Diagnostic challenges of neuropathic tooth pain. J Can Dent Assoc. 2004;70(8):542-546.

7. Peterson LJ, Ellis E, Hupp JR, Tucker MR. Contemporary Oral and Maxillofacial Surgery. Louis, MO: Mosby; 2003.

8. Levin LG, Law AS, Holland GR, Abbott PV, Roda RS. Identify and define all diagnostic terms for pulpal health and disease states. J Endod. 2009;35(12):1645-1657. doi:10.1016/j.joen.2009.09.032.

9. Chamani G, Zarei MR, Mehrabani M, Taghiabadi Y. Evaluation of effects of Zingiber officinale on salivation in rats. Acta Med Iran. 2011;49(6):336-340.

10. Neville B, Damm DD, Allen C, Bouquot J. Epithelial Pathology Oral and Maxillofacial Pathology. 3rd ed. St. Louis: Elsevier; 2009:345.

11. Onwuekwe IO, Onodugo OD, Ezeala-Adikaibe B, et al. Pattern and presentation of epilepsy in Nigerian Africans: a study of trends in the southeast. Trans R Soc Trop Med Hyg. 2009;103(8):785-789. doi:10.1016/j.trstmh.2009.04.010.

12. Headache Classification Subcommittee of the International Headache Society. The International Classification of Headache Disorders: 2nd ed. Cephalalgia. 2004;24 Suppl 1:9-160.

13. Frost D, Hersh E, Levin L. Fonseca Oral and Maxillofacial
Surgery. Philadelphia: Saunders; 2000.

14. Springhouse C. Professional's Handbook of Drug Therapy in Pain Management. Lippincott Williams \& Wilkins; 2001.

15. Weiner RS. Pain Management: A Practical Guide for Clinicians. Boca Raton, IL: CRC press; 2001.

16. Lewis PJ, Pollina J Jr. Neurosurgery Views. Neurosurgery. 2005;2(1).

17. Antipa C, Moldoveanu V, Rusca N, Bruckner II, Podoleanu AG, Stanciulescu V, eds. Low-energy laser treatment of rheumatic diseases: a long-term study. Proc SPIE. 1995. https://www.spiedigitallibrary.org/conferenceproceedings-of-spie/2391/1/Low-energy-laser-treatmentof-rheumatic-diseases--a-long/10.1117/12.209936. short? $\mathrm{SSO}=1$

18. Sweetman SC. Martindale: the complete drug reference: Pharmaceutical press; 2009.

19. Brondon P, Stadler I, Lanzafame RJ. Melanin density affects photobiomodulation outcomes in cell culture. Photomed Laser Surg. 2007;25(3):144-149. doi:10.1089/ pho.2007.2045.

20. Antipa C, Pascu M-L, Pascu R, Ionescu E, eds. Objective methods in evaluating low-level laser therapy results. Proc SPIE. 2001. https://www.spiedigitallibrary.org/ conference-proceedings-of-spie/4606/1/Objectivemethods-in-evaluating-low-level-laser-therapyresults/10.1117/12.446709.short.

21. Ohno T. [Pain suppressive effect of low power laser irradiation. A quantitative analysis of substance $\mathrm{P}$ in the rat spinal dorsal root ganglion]. Nihon Ika Daigaku Zasshi. 1997;64(5):395-400.

22. Asnaashari M, Safavi N. Application of low level lasers in dentistry (endodontic). J Lasers Med Sci. 2013;4(2):57.

23. Amanat D, Ebrahimi H, Lavaee F, Alipour A. The adjunct therapeutic effect of lasers with medication in the management of orofacial pain: double blind randomized controlled trial. Photomed Laser Surg. 2013;31(10):474479. doi:10.1089/pho.2013.3555.

24. Öz S, Gokcen-Rohlig B, Saruhanoglu A, Tuncer EB. Management of myofascial pain: low-level laser therapy versus occlusal splints. J Craniofac Surg. 2010;21(6):17221728. doi:10.1097/SCS.0b013e3181f3c76c.

25. Dundar U, Evcik D, Samli F, Pusak H, Kavuncu V. The effect of gallium arsenide aluminum laser therapy in the management of cervical myofascial pain syndrome: a double blind, placebo-controlled study. Clin Rheumatol. 2007;26(6):930-934. doi:10.1007/s10067-006-0438-4.

26. Altan L, Bingol U, Aykac M, Yurtkuran M. Investigation of the effect of GaAs laser therapy on cervical myofascial pain syndrome. Rheumatol Int. 2005;25(1):23-27. doi:10.1007/ s00296-003-0396-y.

27. Hansen HJ, Thoroe U. Low power laser biostimulation of chronic oro-facial pain. A double-blind placebo controlled cross-over study in 40 patients. Pain. 1990;43(2):169-179.

28. Fulop AM, Dhimmer S, Deluca JR, et al. A meta-analysis of the efficacy of laser phototherapy on pain relief. Clin J Pain. 2010;26(8):729-736. doi:10.1097/AJP.0b013e3181f09713.

29. Carrasco TG, Guerisoli LD, Guerisoli DM, Mazzetto MO. Evaluation of low intensity laser therapy in myofascial pain syndrome. Cranio. 2009;27(4):243-247. doi:10.1179/ crn.2009.035. 
30. Gholami GA, Fekrazad R, Esmaiel-Nejad A, Kalhori KA. An evaluation of the occluding effects of Er;Cr:YSGG, Nd:YAG, $\mathrm{CO}(2)$ and diode lasers on dentinal tubules: a scanning electron microscope in vitro study. Photomed Laser Surg. 2011;29(2):115-121. doi:10.1089/pho.2009.2628.

31. Shirani AM, Gutknecht N, Taghizadeh M, Mir M. Low-level laser therapy and myofacial pain dysfunction syndrome: a randomized controlled clinical trial. Lasers Med Sci. 2009;24(5):715-720. doi:10.1007/s10103-008-0624-5.

32. Hakgüder A, Birtane M, Gurcan S, Kokino S, Turan FN. Efficacy of low level laser therapy in myofascial pain syndrome: an algometric and thermographic evaluation. Lasers Surg Med. 2003;33(5):339-343. doi:10.1002/ lsm.10241

33. Walker J. Relief from chronic pain by low power laser irradiation. Neurosci Lett. 1983;43(2-3):339-344.

34. Yang HW, Huang YF. Treatment of persistent idiopathic facial pain (PIFP) with a low-level energy diode laser.
Photomed Laser Surg. 2011;29(10):707-710. doi:10.1089/ pho.2011.3030.

35. Ilbuldu E, Cakmak A, Disci R, Aydin R. Comparison of laser, dry needling, and placebo laser treatments in myofascial pain syndrome. Photomed Laser Surg. 2004;22(4):306-311. doi:10.1089/pho.2004.22.306.

36. Pinheiro AL, Cavalcanti ET, Pinheiro TI, et al. Low-level laser therapy is an important tool to treat disorders of the maxillofacial region. J Clin Laser Med Surg. 1998;16(4):223226. doi:10.1089/clm.1998.16.223.

37. Pinheiro AL, Cavalcanti ET, Pinheiro TI, Alves MJ, Manzi CT. Low-level laser therapy in the management of disorders of the maxillofacial region. J Clin Laser Med Surg. 1997;15(4):181-183. doi:10.1089/clm.1997.15.181.

38. Gam AN, Thorsen H, Lonnberg F. The effect of low-level laser therapy on musculoskeletal pain: a meta-analysis. Pain. 1993;52(1):63-66. 